\section{Literatur:}

BLAND, N. / MUNDY, G. / RUSSELL, J. / TUFFIN, R. (1999) ,Career Progression of Ethnic Minority Officers', Home Office Research Development and Statistics Directorate, London.

COKER, S. (2005) ,Akzeptanz von dunkelhäutigen Polizeibeamtinnen und Polizeibeamten in Deutschland'. Diplomarbeit zur Erlangung des Grades Diplom-Verwaltungswirt (FH), Hochschule Polizei Baden-Württemberg VillingenSchwenningen.

HARDT, O. (2006) »Black Deutschland « (Dokumentarfilm) Arte-Themenabend »Schwarze Haut, weiße Angst« (http://www.arte-tv.com).
KERSTEN, J. (2006) ,Französische Verhältnisse', KKP-aktuell (Heft 1/Februar), pp. 3-4 (LKA Baden-Württemberg).

KERSTEN, J. (2006) ,Youth Groupings, Identity, and the Political Context - On the Significance of Extremist Youth Groupings in Unified Germany', Gangs in the Global City, hrsg.von J. Hagedorn. University of Illinois Press 2006, pp. 366-401.

MAGUIRE, E. / KING, W. (2004), Trends in the Policing Industry' The Annals of the American Academy of Political and Social Science 593 (May), pp. 15-41.
SKOGAN, W. /MEARES, T. (2004) ,Lawful Policing', The Annals of the American Academy of Political and Social Science 593 (May), pp. 66-83.

SWOBODA-RIECKEN, S. (2001) ,Berufliche Sozialisation und Rollenverständnis der Geschlechter in der Gegenwart. Dargestellt am Beispiel von Frauen in der Schutzpolizei', Dissertation zur Erlangung des Doktorgrades der Erziehungswissenschaftlichen Fakultät der Christian-Albrechts-Universität, Kiel.

ZHAO, J./LOVRICH, N. (1998), Determinants of Minority Employment in American Municipal Police Agencies: The Representation of African American Officers' Journal of Criminal Justice 26 (Nr. 4), pp. 267-277.

\title{
Grundrechte und Ermittlungsverfahren ${ }^{1}$
}

\author{
Ursula Nelles
}

\section{Einleitung}

In der Theorie ist die Strafprozessordnung ein ausbalanciertes Geflecht von Rechten und Pflichten mit rechtsstaatlichen Sicherungen, die auf dem Prinzip der Gewaltentrennung und wechselseitiger Kontrolle beruhen, mit einem Ermittlungsverfahren, das mit einem Anfangsverdacht beginnt und als geheimes Inquisitionsverfahren notwendig und solange erträglich ist, wie seine Ergebnisse Gegenstand eines öffentlichen, mündlichen und justizförmigen Hauptverfahrens sind usw.

Die Fakten stimmen mit der Theorie allerdings nicht immer überein. So zeichnen sich die Aufweichung schon der normativen Grenzen zwischen Strafverfolgung und Prävention und die tatsächliche Entwicklung von repressiver hin zu vorbeugender, um nicht zu sagen pro-aktiver Verbrechensbekämpfung ab. Auch machen Strafverfahren, die mit einem Urteil enden, das auf dem Inbegriff der in mündlicher Verhandlung erhobenen Beweise und ihrer Würdigung beruht und der Wiederaufnahme überhaupt zugänglich ist, nur noch einen verschwindend geringen Bruchteil aller Strafverfahren aus.

Es ist ein offenes Geheimnis, dass diese Entwicklung sich in den vergangenen 10 Jahren verfestigt und beschleunigt hat - auch, aber nicht nur - unter dem Einfluss der europäischen Kriminalpolitik und ihrer Umsetzung in Richtlinien und Rahmenbeschlüssen.

Dieser Beitrag soll deshalb mit Akzentsetzung auf dem Ermittlungsverfahren die betreffende Entwicklung aus der Perspektive des Bürgers als dem primären Adressaten des Strafrechts und seines Vollzugs durch Verfahren beleuchten. Der Beitrag beschränkt sich nicht auf Grundrechte im Ermittlungsverfahren, sondern trägt den Titel
Grundrechte und Ermittlungsverfahren, weil um eine These vorwegzunehmen - verschiedene und sich wechselseitig verstärkende Entwicklungslinien die normativen Grenzen staatlicher Verbrechensbekämpfung in einer Weise erodiert haben, die mit einer Fokussierung nur der je einzelnen Verfolgungsmaßnahme und der Frage nach dem dagegen möglichen Rechtsschutz nicht mehr angemessen erfasst werden kann. Es stellt sich nämlich heraus, dass

- die Sicherungen des Anfangsverdachts nicht mehr funktionieren,

- das Trennungsgebot, das nach überkommenem Verständnis die Grenzen zum Verfassungsschutz beherrscht, aufgeweicht wird,

- die Gewaltenteilung, wie sie etwa im Richtervorbehalt zum Ausdruck kommt, normtechnisch und tatsächlich leerläuft,

- die materiellen Voraussetzungen von Grundrechtseingriffen durch bloße Benutzung des richtigen Formulars ersetzt werden,

- die analoge Anwendung von Eingriffsbefugnissen zum Stand der Technik geworden ist,

- die bislang schon zu beobachtenden apokryphen Haftgründe auch auf andere Sicherungsmaßnahmen übergreifen,

- Beweismittel ohne Rücksicht auf den normativen Kontext der Beweisgewinnung und ihrer Funktionalität in das Strafverfahren transferiert werden und

- die Herren des Verfahrens es selbst in der Hand haben, richterliche Kontrolle ihrer auch verfahrensbeendenden Entscheidungen auszuhebeln oder faktisch zu unterlaufen.

Unter dem Eindruck dieser Defizite muss die Frage gestellt werden, ob nicht ein von solchen Prinzipien geleitetes Ermittlungsverfahren als Ganzes zu einer latenten Verletzung von Grundrechten und $\mathrm{zu}$ einer rechtsstaatlichen Bedro- hung geworden ist. Sowie man im Kontext von Art. 3 GG zwischen offenen und strukturellen Diskriminierungen unterscheiden kann, so kann und muss man auch das Ermittlungsverfahren unter dem Aspekt analysieren, ob es seiner normativen und faktischen Struktur nach Grundrechtsschutz und die vielbeschworenen Bedürfnisse einer effektiven Strafverfolgung in ein angemessenes Verhältnis bringt oder ob es in toto auf strukturelle Grundrechtsfeindlichkeit angelegt und damit rechtsstaatswidrig ist. Das Ergebnis einer solchen Gesamtanalyse sind dann möglicherweise nicht mehr nur Forderungen nach der Korrektur einzelner Eingriffskompetenzen oder Vorschläge für punktuelle Verbesserungen des Rechtsschutzes, sondern es könnte die Notwendigkeit einer Generalrevision nach sich ziehen.

\section{Analyse der strukturellen Rechtsstaats- defizite}

Im Folgenden wird nicht allen Ansatzpunkten nachgegangen, die in der Liste der möglichen Defizite aufgezählt wurden. Einige von ihnen sind bereits so häufig behandelt und beklagt worden, dass man sich partiell auf eine Wiederholung des Meinungsbildes beschränken kann.

\section{Der Anfangsverdacht und das Ende des Verdachts}

Jede staatliche Institution hat in einem System, das auf Arbeitsteilung angelegt ist, eine bestimmte Aufgabe zu erfüllen. Mit dieser Kompetenzzuweisung ist die Ermächtigung der Institution verbunden, sich mit der zugewiesenen Aufgabe zu befassen, zugleich aber auch die Beschränkung, die Erfüllung anderer Aufgaben zu unterlassen. Das ist ein allgemeiner Grundsatz des Staatsorga- 
nisations- und Verwaltungsrechts. Die Strafprozessordnung weist der Staatsanwaltschaft die Aufgabe der Sachverhaltserforschung und die Entschließung darüber zu, ob die öffentliche Klage zu erheben ist ( $\$ 160$ Abs. 1 StPO). Das Ermittlungsverfahren als ganzes ist Gegenstand der Befugnisse der Staatsanwaltschaft, ihre Kompetenz also. Diese beginnt mit dem so genannten Anfangsverdacht, der freilich eine weiche Grenze ist: Es wird zwar immer wieder betont, dass tatsächliche Anhaltspunkte vorliegen müssen, die die Beteiligung des Betroffenen an einer verfolgbaren strafbaren Handlung als möglich erscheinen lassen; indessen handelt es sich im Kern um eine Schlussfolgerung auf der Grundlage kriminalistischer Erfahrung und einer vorläufigen Subsumtion des mutmaßlichen Gesamtsachverhalts unter einschlägige Strafgesetze, die sich im Kopf des Ermittelnden abspielt. Es ist von untergeordneter Bedeutung, ob man das als Beurteilungsspielraum bezeichnet oder nicht. Die Begriffe »Beurteilungsspielraum « oder »Ermessen«, die aus der Dogmatik des öffentlichen Rechts in die StPO transportiert wurden, haben nämlich nur dann einen Sinn, wenn es eine Kontrollinstanz gibt, die solche Entscheidungen überprüfen kann, denn die Funktion dieser Begriffe besteht in der $\mathrm{Ab}$ grenzung des jeweiligen Definitions- und Entscheidungsprimats von Behörde und kontrollierendem Gericht. Eine gerichtliche Überprüfung der Entscheidung der Staatsanwaltschaft, ein Ermittlungsverfahren einzuleiten, sieht die Strafprozessordnung aber für die Dauer des Ermittlungsverfahrens nicht vor, wenn man von dem praktisch bedeutungslosen Klageerzwingungsverfahren absieht, das zudem lediglich das Unterlassen von Ermittlung und öffentlicher Klage zum Gegenstand hat. Die umgekehrte Variante der womöglich willkürlichen - Annahme eines Anfangsverdachts oder der Durchführung von Ermittlungen auf bloße Vermutungen hin ist allenfalls post festum auf dem ebenso bedeutungslosen Weg einer Strafanzeige gegen den Ermittlungsbeamten wegen Verfolgung Unschuldiger oder Falschverdächtigung möglich. Wenn es gleichwohl Judikate zur Zulässigkeit von Vorermittlungen gibt, dann beruhen diese darauf - wie etwa ein Beschluss des $L G$ Offenburg ${ }^{2}$-, dass die Staatsanwaltschaft Beschwerde gegen die Verweigerung einer von ihr im Zuge von Vorermittlungen beantragten richterlichen Vernehmung einlegen kann. Es handelt sich also insoweit um Inzidentprüfungen aus Anlass von Rechtsmitteln gegen einzelne Maßnahmen.

Die StPO sieht auch kein »Einstellungserzwingungsverfahren « vor, weder für die Einstellung nach $§ 170$ Abs. 2 StPO mit dem Inhalt, dass die Ermittlungen keinen für die Erhebung der öffentlichen Klage hinreichenden Tatverdacht ergeben haben, noch für die Einstellung aus Opportunitätsgründen. Hierauf wird an späterer Stelle noch zurückzukommen sein.
Die Durchführung und Fortsetzung eines Ermittlungsverfahrens notfalls bis zum Eintritt der Verfolgungsverjährung muss sich der Beschuldigte also ohne Aussicht auf Rechtsschutz gefallen lassen.

\section{Das Trennungsgebot}

Von daher verwundert es nicht, dass zunächst das Polizeirecht und seit einiger Zeit auch das Recht der Nachrichtendienste in die offene Flanke des Anfangsverdachts gestoßen sind. Die Grenzen des Strafrechts und des Polizeirechts sind aufgelöst und - wie Prantl es formulierte - in ein einheitliches Recht der inneren Sicherheit überführt worden, »das nicht mehr unterscheidet zwischen Schuldigen und Unschuldigen, das keine Verdächtigen oder Unverdächtigen mehr kennt, sondern nur noch potentielle Störer. «3

\section{a) Staatsanwaltschaft / Polizei}

Der Mechanismus ist bekannt; er heißt »vorbeugende Kriminalitätsbekämpfung «, ein Euphemismus, der darüber hinwegtäuscht, dass es um die Einführung gesetzlicher Ermächtigungen für die Polizeibehörden des Bundes und der Länder einschließlich des Grenzschutzes geht, im Namen der inneren Sicherheit verdachtsunabhängige Kontrollen durchzuführen. Nicht nur Verteidiger beklagen öffentlich, dass immer mehr Strafverfahren mit einem Vermerk zum Anfangsverdacht beginnen, der damit eingeleitet wird, dass folgender Sachverhalt »dienstlich in Erfahrung gebracht worden « sei..., dass Ermittlungen ohne jede aktenkundige Tatsachengrundlage eingeleitet werden oder einfach mit einem Bericht der Polizei, der Finanzämter, von Bundesaufsichtsämtern oder anderen Behörden beginnt, die das Produkt ihrer eigenen Ermittlungen an die Staatsanwaltschaft übergeben.

Dass dies möglich ist, hängt freilich nicht nur mit der Unüberprüfbarkeit des Anfangsverdachts zusammen, sondern auch mit der höchstrichterlichen Rechtsprechung und den Gesetzesänderungen der letzten Jahre. So befand der $B G H$ schon 1991, dass die Ergebnisse länger dauernder heimlicher Video-Überwachungen auf der Grundlage polizeigesetzlicher Ermächtigung im Strafverfahren selbst dann verwertbar sind, wenn - wie es damals noch der Fall war - die Strafprozessordnung eine solche Ermittlungsmaßnahme gerade nicht kennt. ${ }^{4}$ Damit hatte die ehemalige Bundesministerin der Justiz Leutheusser-Schnarrenberger schon bei ihrer Ernennung im Jahr 1992 den Kampf gegen den großen Lauschangriff verloren, der über die Polizeigesetze längst Einzug in das Strafverfahren genommen hatte.

Inzwischen ist die Überlappung von repressiven Befugnissen der Staatsanwaltschaft und präventiven Befugnissen der Polizei gesetzlich fest- geschrieben. Das gilt etwa für den Datenabgleich, die so genannte Rasterfahndung, die als repressive Maßnahme in $§ \S 98$ a - c StPO zugelassen ist, aber zugleich als präventive Maßnahme in nahezu allen Polizeigesetzen unter deutlich weiter gefassten Voraussetzungen vorgesehen ist. Das reicht sogar bis in den privatwirtschaftlichen Bereich; Telekommunikationsanbieter müssen aus Gründen ordnungsgemäßer Buchführung die Verbindungsdaten ihrer Kunden über einen längeren Zeitraum speichern, weil diese die Grundlage für die Berechnung des Entgelts für die Nutzung von fremdbetriebenen Netzen bilden. Sie sind nun seit 2001 verpflichtet »unverzüglich Auskunft über Telekommunikationsverbindungsdaten zu erteilen, soweit die Auskunft für die Untersuchung erforderlich ist.« (§ 100g Abs. 1 S. 1). Da sich diese Auskunftspflicht auch auf »zukünftige Telekommunikationsverbindungen « (§ 100g Abs. 1 S. 2) beziehen kann, hat das zu Folge, dass sämtliche Verbindungsdaten (nicht allerdings der Inhalt der Gespräche) aller Bürger der Bundesrepublik beständig überwacht und gespeichert werden. ${ }^{5}$

Auf den durch § 100i StPO zugelassenen Einsatz von so genannten IMSI-Catchern ${ }^{6}$ sei nur am Rande hingewiesen. Diese Geräte simulieren die Basisstation eines Mobilfunknetzes und fangen damit jedes eingeschaltete Handy im Empfangsbereich ab. Damit sind Bewegungsprofile aber auch das Abhören von Gesprächen möglich. ${ }^{7}$

Was die grundsätzliche Kritik an dieser Entwicklung angeht, sei auf die Generalabrechnung mit dieser Entwicklung durch Frehsee ${ }^{8}$ verwiesen.

\section{b) Verbund Nachrichtendienste/Staatsanwalt- schaft / Polizei}

Inzwischen hat sich diese gesetzgeberische Methode der Grenzverwischungen zwischen den Kompetenzen der Präventivbehörden und der Strafverfolgungsbehörden auch auf die Nachrichtendienste ausgedehnt. Durch das StrafverfahrensänderungsG 1999 sind im Jahre 2000 aus vorgeblich datenschutzrechtlichen Gründen Regelungen in die StPO eingestellt worden, die die Weitergabe von Erkenntnissen aus Strafverfahren an die Nachrichtendienste regeln ( $\$ 474$ Abs. 2 StPO). Dabei handelt es sich im Wesentlichen um die Weitergabe von Informationen mit Bezug zu Staatsschutzdelikten. Über die umgekehrte Richtung sagt die StPO nichts aus, wenn man von dem allgemeinen Auskunftsanspruch der Staatsanwaltschaft nach $\S 161$ Abs. 1 StPO absieht. Insoweit sehen aber die Gesetze über Nachrichtendienste (§§ 19 Abs. 1 S. 1 BundesverfassungsschutzG, 11 Abs. 1 MAD-G und 9 Abs. 1 S. 1 BND-G) im Wesentlichen gleichlautende Spezialreglungen vor, die teils aufeinander Bezug nehmen und durch das TerrorismusbekämpfungsG v. 9. 1. 2002 einander angepasst wurden. Danach dürfen die Nachrichtendienste personenbezogene Daten an inländische Behörden übermitteln, wenn 
dies zur Erfüllung ihrer jeweiligen Aufgabe erforderlich ist »oder der Empfänger die Daten zum Schutz der freiheitlich demokratischen Grundordnung oder sonst für Zwecke der öffentlichen Sicherheit benötigt.«

Die Justiz- und Innenminister der Länder haben darauf ihrerseits mit einer Anpassung der RiStBV reagiert, die das Verfahren der Übermittlung regeln. Aber in Nr. 205 Abs. 5 gehen sie darüber hinaus: Angehörige der Behörden für Verfassungsschutz sollen als Auskunftspersonen zu Ermittlungshandlungen der Staatsanwaltschaft wie z. B. zu den ausdrücklich genannten Tatortbesichtigungen, Durchsuchungen oder Beschlagnahmen hinzugezogen werden können. Ferner ist nach Abs. 6 der unmittelbare Geschäftsverkehr der StA mit den Verfassungsschutzbehörden zulässig, und der unmittelbare Durchfluss aller Informationen ist damit hergestellt.

Das Pikante an dieser Art des Umgangs mit dem Gesetz und seiner Interpretation in Gestalt genereller Weisungen - mehr sind die RiStBV nach wie vor nicht - ist, dass die gesetzlichen Regelungen den aus dem Recht auf informationelle Selbstbestimmung abgeleiteten Vorgaben des Bundesverfassungsgerichts ${ }^{9}$ Rechnung tragen wollten. Danach reicht insbesondere eine nur verwaltungsinterne Ermächtigung zur Datensammlung, -speicherung und -übermittlung nicht aus. Kaum aber ist die erforderliche gesetzliche Ermächtigung geschaffen, beginnt die Exekutive bereits wieder damit, ihre Befugnisse nach dem Prinzip der Grenzmoral in die Grauzone zu überdehnen.

Expressis verbis setzt sich der Generalbundesanwalt ${ }^{10}$ für den Verbund von Nachrichtendiensten und Strafverfolgungsbehörden ein. Sub specie Terrorismusbekämpfung fordert er unter Berufung auf den von ihm geprägten Begriff der »neuen Sicherheitsarchitektur « einen "ganzheitlichen Bekämpfungsansatz « und verdeutlicht den »Stellenwert der informationellen Zusammenarbeit der Sicherheitsbehörden untereinander und mit der Staatsanwaltschaft ... eindringlich «. - Erforderlich sei »ein abgestimmtes Vorgehen aller staatlichen Stellen, um Strukturen, Logistik und finanzielle Ressourcen erkennen und terroristische Vorhaben vereiteln zu können.« Die eigentlich alarmierende Information in diesem Artikel ist für mich allerdings, dass »ein zentrales Lage- und Analysezentrum« »im Gespräch« ist.

Das einzige Problem, das Nehm für die »faktische Herausbildung nachrichtendienstlicher Strukturen in Kombination mit polizei-imperativen Befugnissen « sieht, ist das Trennungsgebot, das der Entstehung eines allmächtigen Überwachungsstaates entgegensteht. Er bemüht sich deshalb um den Nachweis, dass dieses Gebot keinen Verfassungsrang habe und daher durch einfachgesetzliche Regelungen derogiert werden könne. Dazu greift er auf die Entstehungsgeschichte zu- rück, den so genannten Polizeibrief der Alliierten Militärgouverneure an den Parlamentarischen Rat, der die Ermächtigung zur Einrichtung von Nachrichtendiensten unter den Vorbehalt stellte, dass diese Stelle keine Polizeibefugnisse haben sollte. Weil, so seine Argumentation, die darauf beruhende Fassung des Art. 87 Abs. 1 S. 2 GG lediglich Auskunft über die Aufgaben der Sicherheitsbehörden gebe und aus der Aufgabenzuweisung nicht auf die Befugnisse zur Aufgabenbewältigung geschlossen werden könne, schließe das Grundgesetz nicht aus, den Nachrichtendiensten bestimmte Befugnisse vorzuenthalten. Jedenfalls habe der Polizeibrief seit Wiedererlangung der Souveränität Deutschlands keinen Verfassungsrang mehr. Es handele sich bei dem Trennungsgebot lediglich um ein besonderes Organisationsprinzip, dem sich die demokratischen Kräfte übereinstimmend verpflichtet fühlten ähnlich - »unverbindlich « muss man wohl hinzufügen - wie der Verpflichtung zur Wiedergutmachung nationalsozialistischen Unrechts. Weil das geltende Recht nun einmal eine Überlagerung der Aufgaben vorsehe, sei es rechtlich auch unproblematisch, wenn die Behörden, die aus eigenem Recht gleichzeitig und unabhängig voneinander identische Aufklärungen betreiben könnten, dies sogleich gemeinsam täten. Danach sei selbst der Informationsaustausch einschließlich der Weitergabe von unter Zwang gewonnenen Erkenntnissen aus einem Strafverfahren unproblematisch.

Jedes der Argumente auf seine mangelnde Schlüssigkeit hin abzuklopfen, würde den Rahmen dieses Beitrages übersteigen. Daher soll die Feststellung genügen, dass ein fundamentales Organisationsprinzip eines rechtsstaatlichen Gemeinwesens, nämlich dass jede Institution sich an die Grenzen ihrer Befugnisse zu halten hat, hier mit dem Postulat beiseite gefegt wird, dass der Zweck die Mittel heiligt.

Als 1956 die Kommunistische Partei Deutschlands verboten worden war und zehntausende von Strafverfahren gegen vermeintliche Kommunisten geführt wurden, war es ausgerechnet ein Staatsanwalt, der öffentlich Bedenken äußerte. Richard Schmid, Generalstaatsanwalt Stuttgart, sah das »Wesen einer Diktatur « darin, dass sie die Abwehr von ihr feindlichen Tendenzen weit nach vorn verlagere, nämlich in »Rechtssphären, die in einem freien Staat durch Individualrechte gesichert « seien. Diejenige Staatsform sei »die vollkommenste Diktatur, die diese Vorverlegungen am vollkommensten « zustande bringe. Daran erinnert Prantl in dem bereits zitierten Buch und stellt fest, dass derzeit im Westen ein »Wettstreit um die vollkommenste Diktatur « ausgebrochen sei. ${ }^{11}$ Dem kann nur zugestimmt werden.

Symptomatisch dafür ist eine Diskussion, die im Europäischen Parlament vor einiger Zeit geführt wurde: Die britische Ratspräsidentschaft hatte den Vorschlag eingebracht, alle Daten aus Telefonverbindungen und Emails für mindestens ein Jahr aufzuheben, damit sie für etwaige Ermittlungen gegen mögliche Terroristen verfügbar sind. Der zuständige FDP-Abgeordnete Alexander Alvaro hat die Ablehnung dieses Projekts durch den zuständigen Ausschuss für bürgerliche Freiheiten wie folgt begründet: »Jeder Erstsemesterstudent in Jura lernt, dass ein Grundrechtseingriff einer Rechtfertigung bedarf. Solange die nicht vorliegt, ist er rechtswidrig. Damit fliegt man in jeder Klausur durch, nur im Ministerrat nicht! « Das Parlament ist ihm gefolgt, allerdings nur mit der Konsequenz, dass der Kommissar für Justiz und Inneres, Franco Frattini, einen Kompromissvorschlag angekündigt hat, dem zufolge nur Telefondaten ein Jahr lang gespeichert werden sollen, Emails dagegen nur für ein halbes Jahr. ${ }^{12}$

\section{Präventive und nachträgliche richterliche Kontrolle als Ausgleich?}

Nur ganz kurz sei auf die altbekannte Frage eingegangen, ob der Richtervorbehalt, unter den die Strafprozessordnung jedenfalls die schwerwiegenden Grundrechtseingriffe stellt, als rechtsstaatlich hinreichende Remedur gelten kann. Das Ergebnis ist schnell ermittelt: Der Richtervorbehalt läuft leer.

Soweit der Staatsanwaltschaft und ihren Hilfsbeamten Ausnahmekompetenzen bei Gefahr im Verzug zustehen, machen sie davon gern Gebrauch, wie die zahlreichen Entscheidungen des Bundesverfassungsgerichts ${ }^{13}$ belegen, das nicht müde wird, der Staatsanwaltschaft die Verpflichtung vor Augen zu führen, die vorgängige Entscheidung des Ermittlungsrichters einzuholen, und die Gerichte anzuhalten, ihrer Kontrollpflicht auch durch geeignete organisatorische Vorkehrungen nachzukommen.

Aber auch wenn sich alle daran halten, kann von effektiver Kontrolle kaum die Rede sein. Das hat eine Bielefelder Studie zur Anordnungspraxis bei der Telefonüberwachung ${ }^{14}$ ergeben: In weit über $90 \%$ der Fälle haben die Richter den mit dem Antrag der Staatsanwaltschaft überreichten Beschlussentwurf wortwörtlich übernommen. ${ }^{15}$

Das kann und wird ganz gewiss nicht anders aussehen, wenn es - wie in den polizeigesetzlichen und nachrichtendienstlichen Vorschriften vorgesehen - der Behördenleiter ist, der über die Anordnung der Maßnahmen entscheidet.

Soweit eine nachträgliche Anrufung des Richters nach dem Muster des $\S 98$ Ab. 2 S. 2 StPO vorgesehen ist, dürfte diese noch aus anderen Gründen ins Leere laufen: die modernen Ermächtigungsgrundlagen erkennt man nicht nur daran, dass sie sehr lang und handwerklich schlecht gemacht sind, sondern auch daran, dass es sich um Normen handelt, die nur scheinbar über ei- 
nen Tatbestand verfügen. Auf der Voraussetzungsseite findet man, freundlich gesprochen: unbestimmte Rechtsbegriffe, realistisch betrachtet: leere Worthülsen, die nur mühsam verdecken, dass es um blanke Zweckmäßigkeit geht. Da das gesamte Ermittlungsverfahren der Gestaltungsfreiheit unterliegt, denn z. B. Telefonüberwachungen müssen nicht, sondern können angeordnet werden, dominiert das Ermessen auf der Rechtsfolgenseite und als rechtliche Grenze bleibt einzig der Grundsatz der Verhältnismäßigkeit, der seine Funktion aber kaum noch erfüllen kann, wenn die Mittel-Zweck-Relation mit so schwammigen Kriterien auszufüllen ist wie: »Straftaten von erheblicher Bedeutung « oder »wenn die Erforschung des Sachverhalts auf andere Weise weniger erfolgversprechend oder wesentlich erschwert wäre«. Wo Normen keine Grenzen mehr setzen, können Richter auch die Einhaltung von Grenzen nicht mehr kontrollieren.

Aber auch diejenigen Normen, die vermeintlich noch klar sind, weil sie - wie etwa die Telefonüberwachung - an einen fest umrissenen Katalog von Anlasstaten anknüpfen, sind keine wirkliche Hilfe. Der Katalog des § 100a StPO ist von seiner Einführung im Jahre 1968 bis zum Jahr 2001 insgesamt 20 mal geändert worden; dabei handelte es sich in 18 Fällen um eine Ausweitung des Katalogs. ${ }^{16}$ Die Fernmeldeüberwachung ist durch die ständigen Erweiterungen von der Ausnahme zum Normalfall für eine fast unübersehbare Fülle unterschiedlichster Deliktsfälle geworden. Der Versuch einer Einschränkung mit Hilfe von Katalogen muss als gescheitert angesehen werden. ${ }^{17}$

\section{Ersatz von materiellen Eingriffsvorausset- zungen durch Benutzung des richtigen Formulars}

In diese Entwicklung der Entleerung von Eingriffsnormen zu mehr oder weniger voraussetzungslosen Ermächtigungen fügt sich auch der Rahmenbeschluss über den Europäischen Haftbefehl ein. Das deutsche Gesetz über den europäischen Haftbefehl ist vom Bundesverfassungsgericht für verfassungswidrig erklärt worden. ${ }^{18}$ Die Verpflichtung Deutschlands, ein entsprechendes Gesetz zu erlassen, bleibt aber bestehen. Der Rahmenbeschluss bedient sich des Katalogtatensystems. Allerdings ist der Katalog noch unbestimmter und offener, als die uns im deutschen Recht bekannten, weil die Bezeichnungen der Anlass-Straftaten so abstrakt gefasst sind, dass alle Mitgliedstaaten ihre Strafrechtsordnungen darunter subsumieren können. Die europaweite Vollstreckung eines solchen Haftbefehls hängt dann nur noch davon ab, dass der Ermittlungsrichter das in dem Rahmenbeschluss genau beschriebene und ihm als Anlage beigefügte europäische Formular benutzt.

\section{Ausdehnung von Eingriffsermächtigungen im Wege der Analogie}

Der Wettkampf um die »vollkommenste Diktatur « scheint nun nicht nur den europäischen und die nationalen Gesetzgeber zu Höchstleistungen anzustacheln und den Generalbundesanwalt umzutreiben, sondern stimuliert offenbar auch die kreative Fantasie von Richtern und von Vertretern unserer Zunft, wenn es darum geht, neue Formen und Wege zur Ausforschung des Individuums zu ersinnen. Besonders beliebt ist dabei die Entdeckung von planwidrigen Regelungslücken bekanntlich die erste Voraussetzung für die $\mathrm{Zu}$ lässigkeit analoger Anwendung von Gesetzen.

Zunächst ist die Praxis auf das Problem gestoßen, dass immer weniger Menschen per Telefon kommunizieren und immer mehr Menschen, wenn sie Informationen per Internet austauschen, mit Verschlüsselungen arbeiten. Deswegen hätte man am liebsten den gesamten Inhalt einer Festplatte zur Verfügung, um die Verschlüsselungen zu knacken und die Informationsquelle zum Sprudeln zu bringen. Technisch ist das ganz einfach: Man schleust einen »Trojaner « oder ein so genanntes »Backdoor-Programm « ein. Wenn der Zielrechner in Betrieb und »online « ist, kann der gesamte Datenspeicher mit diesen Programmen gelesen und heruntergeladen werden. Der $B G H$ hat in einem umstrittenen Beschluss ${ }^{19}$ eine solche Maßnahme auf § 100a StPO - Telekommunikationsüberwachung - gestützt, aber nur den einmaligen Zugriff auf die Mailbox gestattet, weil die Maßnahme auch Elemente einer Durchsuchung und Beschlagnahme enthalte und deshalb nicht dauerhaft durchgeführt werden könne.

Hofmann hat diese Methode als »Online-Durchsuchung « bezeichnet und damit dann auch flugs die Analogie zu $\S \S 102,105,94,98$ StPO begründet: Das Trojanische Pferd sei letztlich nur ein elektronisch operierender verdeckter Ermittler, also wie dieser nach $\S 110$ a StPO einsetzbar. Er durchsuche den Computer - nicht etwa in den Grenzen der Fernmeldeüberwachung, sondern unter den einfachen Voraussetzungen für eine Durchsuchung ( $\S \S 102,105$ StPO) - sammele die Informationen und sichte sie nach den Vorschriften über die Durchsicht von Papieren. ${ }^{20}$ Es handele sich also keinesfalls um staatliches Hacking, sondern um eine zulässige Ermittlungsmaßnahme.

Eine weitere Idee wird von Seiten des Polizeirechts beigesteuert. Schieder stellt die These auf, dass die automatisierte Erkennung amtlicher KfzKennzeichen eine zulässige polizeiliche Maßnahme sei, deren Ergebnisse auch gespeichert und für Zwecke von Strafverfahren übermittelt werden dürften. ${ }^{21}$ Gemeint ist damit im Klartext die Videoüberwachung etwa von öffentlichen Parkhäusern auch mit dem Ziel der Sammlung von Kfz-Kennzeichen. Beiläufig wird dabei bereits das rechtliche Feld so bestellt, dass auch die Daten aus den Überwachungsanlagen für die elektronische Berechnung der Mautgebühren dem Zugriff der Polizei und damit mittelbar der Staatsanwaltschaft ausgeliefert sind.

\section{Unbegrenzte Verwertbarkeit; »Prinzip der wechselseitigen Anerkennung «}

Erst seit die EU das Prinzip der wechselseitigen Anerkennung zum Paradigma der grenzüberschreitenden Strafrechtspflege erklärt hat, ist die Diskussion darüber wieder aufgelebt, ob und wie Beweismittel ohne Rücksicht auf den normativen Kontext ihrer Gewinnung und ihrer Funktionalität von einem Strafverfahren in ein anderes oder auch von einem beliebigen Verfahren in das Strafverfahren transferiert werden können. Gless ist in ihrer Habilitationsschrift dieser Frage am Beispiel von drei Rechtsordnungen nachgegangen und hat dabei die Verwerfungen analysiert, die ein voraussetzungsloser Transfer in den jeweiligen Rechtsordnungen gerade mit Blick auf die unterschiedlichen grundrechts- und freiheitssichernden Vorkehrungen der jeweiligen Prozessordnung nach sich zieht.

In Deutschland kennen wir dieses Prinzip seit Langem; das Gerichtsverfassungsgesetz (§§ 156 ff.) regelt es im Hinblick darauf, dass Justiz und Polizeibehörden Ländersache sind und deshalb »grenzüberschreitende « Ermittlungen, Entscheidungen und Vollstreckungen bei uns zum Alltag gehören. Wir haben es aber schleichend auch auf die funktionalen Grenzen zwischen unterschiedlichen Verfahren erweitert: Beweismittel werden nahezu unbegrenzt verschoben, wie sich etwa an dem Konzept des Datenaustausches zwischen Nachrichtendiensten, Polizei und Staatsanwaltschaft ablesen lässt. Hierauf sei allerdings nicht weiter eingegangen, weil ein weiterer großer Entwicklungsstrang wichtiger ist, der im materiellen Strafrecht angelegt ist.

\section{Vorläufige Sicherungen; »apokryphe Grün-} de«

Unter dem Einfluss internationaler Vorgaben, etwa der Financial Action Task Force ${ }^{22}$, die den Ansatz verfolgen, dass die Organisierte Kriminalität ausgetrocknet werden könne, ist auch in Deutschland die Geldwäschegesetzgebung zum Motor für staatliche Vermögensabschöpfung geworden. Die Vorschriften über den erweiterten Verfall, ihre extensive höchstrichterliche Auslegung (Stichwort: Bruttoprinzip), die höchstrichterliche »Infizierungstheorie« zu § 261 StGB, wonach ein einziger Cent gewaschenen Geldes das gesamte Vermögen einer Person zu schmutzigem Geld macht, sind in der Praxis zu einem scharfen Instrument insbesondere in Wirtschaftsstrafverfahren geworden. § 111d StPO lässt nämlich schon im Ermittlungsverfahren den dinglichen Arrest zu, wenn Gründe für die Annahme vorhanden 
sind, dass die Voraussetzungen des Verfalls von Wertersatz oder der Einziehung von Wertersatz vorliegen (111b Abs. 2 StPO).

Aus dem Recht der Untersuchungshaft kennen wir den Begriff der »apokryphen Haftgründe«. Damit ist gemeint, dass Haftbeschlüsse jenseits ihrer formalen Rechtfertigung in Wahrheit durch den Grund motiviert sind, dass die unmittelbare Erfahrung der Haft die Geständnis- oder Kooperationsbereitschaft des Beschuldigten erheblich steigern kann.

Es ist nun zu vermuten, dass das Einfrieren von Vermögen auf Verdacht in der Praxis ebenfalls in dieser Weise dysfunktional eingesetzt wird. Ein viel intensiveres Gebrauchmachen von dem Instrument der Rückgewinnungshilfe (also dem dinglichen Arrest zur Sicherung der Ansprüche von Verletzten) könnte zur Folge haben, dass die Beschuldigten in Wirtschaftsstrafverfahren sich die Vielzahl teurer Strafverteidiger nicht leisten könnten. Es gibt Fälle, in denen das Einfrieren des gesamten Vermögens des Beschuldigten letztlich der einzige Grund dafür war, dass dieser, obwohl er stets seine Unschuld beteuerte und die Beweislage seine These auch stützte, nach steter Verzögerung des Verfahrens durch die Staatsanwaltschaft und später durch das Gericht schließlich einer Einstellung des Verfahrens nach $\S 153 \mathrm{a}$ StPO zustimmte, weil er existentiell am Ende war. Vor diesem Hintergrund klingt § 136a StPO geradezu idyllisch. Die stete Ausdehnung von gesetzlich für zulässig erklärten Eingriffs- und Zwangsmitteln birgt ein solches Drohpotential, dass die Drohung mit und die Anwendung von gesetzlich zulässigem Zwang völlig ausreicht, die Freiheit der Willensentschließung des Beschuldigten zunichte zu machen.

\section{Unkontrollierbarkeit}

Es kommt hinzu, dass es die Herrin des Ermittlungsverfahrens selbst in der Hand hat, ob sogar willkürliche Verfahrensbeendigungen noch richterlich kontrolliert werden können. Als Stichwort seien hier nur Absprachen im Strafprozess genannt. Die höchstrichterliche Rechtsprechung müht sich ebenso redlich wie vergeblich, dem Deal rechtliche Grenzen zu setzen, wie zuletzt noch der Große Senat mit seiner Entscheidung zur Unwirksamkeit eines ausgehandelten Rechtsmittelverzichts. Erreichen kann der $B G H$ nur Absprachen, die urteilsrelevant werden. Verfahrenseinstellungen, die die Zustimmung des Beschuldigten, also die Unterwerfung unter den Zwang, voraussetzen, sind damit nicht mehr erreichbar. Soweit sie im Ermittlungsverfahren durch die Staatsanwaltschaft vorgenommen werden, ist zwar auch die richterliche Zustimmung erforderlich. Es dürfte sich mit der richterlichen Kontrolle der im Einzelfall abgestimmten Voraussetzungen und Bedingungen der Verfahrenseinstellung aber ähnlich verhalten, wie mit der richterlichen
Anordnung von Telekommunikationsüberwachungen nach der Bielefelder Studie: Warum noch den Inhalt überprüfen, wenn doch alle anderen bereits einverstanden sind? Das Zustimmungserfordernis kann also nicht ernsthaft als effektiver Rechtsschutz bezeichnet werden.

\section{Fazit}

Das gesetzliche Ausmaß, die Möglichkeiten der Kumulierung von Überwachungs- und Ausforschungsmitteln im Ermittlungsverfahren hat längst die Grenzen überschritten, die das Bundesverfassungsgericht dem Staat mit Blick auf das Grundrecht der informationellen Selbstbestimmung gezogen hat. Die Intensität der Zwangsmittel, hier insbesondere der staatliche Zugriff auf Vermögen, enthält das Potential für schwere Eingriffe in das Eigentumsrecht und mittelbare Eingriffe in die Freiheit der Berufsausübung, von der allgemeinen Handlungsfreiheit ganz abgesehen, die sich mit dem Grundsatz der Verhältnismäßigkeit nur noch schwer in Einklang bringen lassen. Schließlich gilt noch immer die Unschuldsvermutung. Der Beschuldigte ist, verwaltungsrechtlich gesprochen, allenfalls Anscheinsstörer; im Ermittlungsverfahren ist Grundlage von Eingriffen in seine Grundrechte nur der Verdacht und nicht die vermutete Schuld. So die schöne Theorie, aber sie steht in offenem Widerspruch zu der "neuen Sicherheitsarchitektur «, deren Droh- und Willkürpotential solche Fundamentalfreiheiten nahezu vollständig und gerichtlich kaum noch überprüfbar aushebelt.

Vor diesem Hintergrund mag man kaum noch an die alte These erinnern, dass das Strafverfahrensrecht ein Seismograph für die Rechtsstaatlichkeit eines Gemeinwesens ist. Das Ergebnis ist zu deprimierend. Es sollte aber dazu anspornen, völlig neu darüber nachzudenken, ob überhaupt und wenn ja wie, Strafrecht im klassischen Sinne in die neue Sicherheitsarchitektur so eingepasst werden kann, dass für den einzelnen Beschuldigten noch ein Rest von Grundrechtssicherheit gewährleistet werden kann.

Prof. Dr. Ursula Nelles ist Inhaberin des Lehrstuhls für Strafrecht, Strafprozessrecht und Wirtschaftsstrafrecht an der Westfälischen Wilhelms-Universität Münster.

\section{Fußnoten}

1 Es handelt sich um einen Vortrag, der bei einem Kolloquium aus Anlass der Emeritierung von Jürgen Regge an der Universität Greifswald gehalten wurde, ergänzt um einige Fußnoten und gekürzt um die persönlichen Bezüge.

2 LG Offenburg, Beschluss vom 25.05.1993 Qs 41/ 93, NStZ 1993, $506 \mathrm{ff}$.

3 Heribert Prantl, Verdächtig. Der starke Staat und die Politik der inneren Unsicherheit, 2002, S. 11.

4 BGH, Urteil vom 14.5.1991 - 1 StR 699/90, NStZ 1992, $44 \mathrm{ff}$.
5 Im Einzelnen Welp, GA 2002, 535 ff. 6 International Mobile Subscriber Identification = Kennung des Teilnehmers am Mobilfunkverkehr IMEI (International Mobile Equipment Identification) ist die Gerätekennung.

7 Im Einzelnen dazu Gercke, MMR 2003, $453 \mathrm{ff}$.

8 Frehsee in Frehsee/Löschper/Smaus (Hg), Konstruktion der Wirklichkeit durch Kriminalität und Strafe, 1997, S. $14 \mathrm{ff}$.

9 BVerfGE 65, S. 1 ff.

10 Nehm, NJW 2004, 3289 ff.

11 Prantl, a. a. O., S. 23.

12 EU - Vorerst wohl kein Datenlager, MZ Nr. 225, 39. Woche, Dienstag 27. 9. 2005, S. 2

13 Vgl. nur BVerfG, Urteil vom 20.2. 2001 - 2 BvR 1444/00, NJW 2001, 1121.

14 Backes / Gusy/Begemann/Doka/Finke, Wirksamkeitsbedingungen von Richtervorbehalten bei Telefonüberwachungen (Kurzfassung des Abschlussberichts), Universität Bielefeld 2002. 15 Wie vor, S. $46 \mathrm{ff}$.

16 Niehaus, Katalogtatensysteme als Beschränkung strafprozessualer Eingriffsbefugnisse, 2001, S. $25 \mathrm{ff}$.

17 Niehaus, a.a. O., S. 91 m. w. N.

18 BVerfG, Urteil vom 18. 7. 2005 - 2 BvR 2236/04, NJW 2005, 2289 ff.

19 BGH, Beschluss vom 31.7.95 - 2 BJs 94/94-6 u. a., NJW 1997, $1934 \mathrm{ff}$.

20 Hofmann, NStZ 2005, $121 \mathrm{ff}$.

21 Schieder, NVwZ 2004, $778 \mathrm{ff}$.

22 Die Financial Action Task Force (FATF) ist ein Gremium der internationalen Zusammenarbeit gegen die Geldwäsche. Während des G7-Gipfels in Paris 1989 gegründet, hat sie mittlerweile 33 Mitglieder (zumeist OECD-Staaten sowie den Golfkooperationsrat und die EU). Ihr Sekretariat befindet sich unter dem Dach der OECD. Aufgabe der FATF ist es, Methoden der Geldwäsche aufzudecken, Politikempfehlungen für wirksame Maßnahmen gegen die Geldwäsche zu entwickeln und weltweit dafür zu werben. 